\title{
Response to the Counterexample by K. V. Storozhuk
}

\section{E. Filippov ${ }^{1 *}$ and V. S. Mokeychev ${ }^{1 * *}$}

\author{
(Submitted by A. M. Elizarov) \\ ${ }^{1}$ Institute of Computational Mathematics and Information Technologies, \\ Kazan (Volga Region) Federal University, Kazan, Tatarstan, 420008 Russia \\ Received December 2, 2018; revised January 31, 2019; accepted January 31, 2019
}

\begin{abstract}
We give some explanations and objections to the counterexample to results obtained in our paper [1] proposed in [2].
\end{abstract}

DOI: $10.1134 / \mathrm{S} 199508021905007 \mathrm{X}$

Keywords and phrases: implicit function, continuousness, zeros of functions.

1. The citation is inaccurate. The original assertion is the following one.

Recall that $\tau \in \Omega \subset R^{n}, \xi \in[a, b]$, and $g(\tau, \xi)$ is a continuous real valued function defined on a compact set. Everywhere $g(\tau, a) \cdot g(\tau, b)<0, \xi(\tau)$ is the least root of the equation $g(\tau, \xi)=0$. . . Theorem 2. With each $\varepsilon>0$ there exists a continuous function $g_{\varepsilon} g(\tau, \xi)$ such that $\left|g(\tau, \xi)-g_{\varepsilon}(\tau, \xi)\right|<$ $\varepsilon$, and its least root $\xi(\tau)$ is a sign changing point of the function $g_{\varepsilon}(\tau, \xi)$.

In the paper by K. V. Storozhuk: We give a counterexample to the following assertion from [1]: For every $\varepsilon>0$ and every function $g(\tau, \xi) \in R, \in[a, b]$, continuous on a compact set $\Omega \in R^{n}$ and such that $g(\tau, a) \cdot g(\tau, b)<0$, there exist a function $g_{\varepsilon}(\tau, \xi)$ for which the least root $\xi(\tau)$ of the equation $g_{\varepsilon}(\tau, \xi)=0$ depends continuously on $\tau$ if $\left\|g-g_{\varepsilon}\right\|_{C}<\varepsilon$. Clearly, the distinction goes beyond notations $\left|g(\tau, \xi)-g_{\varepsilon}(\tau, \xi)\right|<\varepsilon$ and $\left\|g-g_{\varepsilon}\right\|_{C}<\varepsilon$.

2. Assume that there exists a continuous function $g(\tau, \xi)$ whose projection is shown in a figure given in our paper. One can easily see that the least zero $\xi(\tau)$ of the function $g(\tau, \xi)$ continuously depends on $\tau$. Therefore we can put $\left.g_{\varepsilon}(\tau, \xi)=g \tau, \xi\right)$, i.e. our proposition is false.

\section{REFERENCES}

1. I. E. Filippov and V. S. Mokeychev, "The least root of a continuous function," Lobachevskii J. Math. 39 (2), 200-203 (2018).

2. K. V. Storozhuk, "On the Article 'The least root of a continuous function'," Lobachevskii J. Math. 39 (9), 1445 (2018).

\footnotetext{
${ }^{*}$ E-mail: Igor.Filippov@kpfu.ru

${ }^{* *}$ E-mail: Valery.Mokeychev@kpfu.ru
} 